\title{
Phosphatase inhibition by LB-100 enhances BMN-111 stimulation of bone growth
}

\author{
Leia C. Shuhaibar, ${ }^{1}$ Nabil Kaci, ${ }^{2,3}$ Jeremy R. Egbert, ${ }^{1}$ Thibault Horville, ${ }^{2}$ Léa Loisay, ${ }^{2}$ Giulia Vigone, ${ }^{1}$ \\ Tracy F. Uliasz, ${ }^{1}$ Emilie Dambroise, ${ }^{2}$ Mark R. Swingle, ${ }^{4}$ Richard E. Honkanen, ${ }^{4}$ Martin Biosse Duplan,,${ }^{2,5}$ \\ Laurinda A. Jaffe, ${ }^{1}$ and Laurence Legeai-Mallet ${ }^{2}$ \\ 'Department of Cell Biology, University of Connecticut Health Center, Farmington Connecticut, USA. ${ }^{2}$ Université de Paris, \\ Imagine Institute, Laboratory of Molecular and Physiopathological Bases of Osteochondrodysplasia, INSERM UMR 1163, \\ F-75015, Paris, France. Inovarion, F-75005 Paris, France. ${ }^{4}$ Department of Biochemistry and Molecular Biology, University of \\ South Alabama, Mobile Alabama, USA. ${ }^{5}$ Service de Médecine Bucco-Dentaire, Hôpital Bretonneau, AP-HP, Paris, France.
}

Activating mutations in fibroblast growth factor receptor 3 (FGFR3) and inactivating mutations in the natriuretic peptide receptor $\mathbf{2}$ (NPR2) guanylyl cyclase both result in decreased production of cyclic GMP in chondrocytes and severe short stature, causing achondroplasia (ACH) and acromesomelic dysplasia, type Maroteaux, respectively. Previously, we showed that an NPR2 agonist BMN-111 (vosoritide) increases bone growth in mice mimicking ACH (Fgfr $3^{\mathrm{Y} 367 \mathrm{Cl}+}$ ). Here, because FGFR3 signaling decreases NPR2 activity by dephosphorylating the NPR2 protein, we tested whether a phosphatase inhibitor (LB-100) could enhance BMN-111-stimulated bone growth in ACH. Measurements of cGMP production in chondrocytes of living tibias, and of NPR2 phosphorylation in primary chondrocytes, showed that LB-100 counteracted FGF-induced dephosphorylation and inactivation of NPR2. In ex vivo experiments with $\mathrm{Fgfr}^{\mathrm{r} 367 \mathrm{C} /+}$ mice, the combination of BMN-111 and LB-100 increased bone length and cartilage area, restored chondrocyte terminal differentiation, and increased the proliferative growth plate area, more than BMN-111 alone. The combination treatment also reduced the abnormal elevation of MAP kinase activity in the growth plate of $\mathrm{Fgfr}^{\mathrm{r} 367 \mathrm{C} /+}$ mice and improved the skull base anomalies. Our results provide a proof of concept that a phosphatase inhibitor could be used together with an NPR2 agonist to enhance cGMP production as a therapy for $\mathrm{ACH}$.

Conflict of interest: The authors have declared that no conflict of interest exists.

Copyright: (c) 2021, Shuhaibar et al. This is an open access article published under the terms of the Creative Commons Attribution 4.0 International License.

Submitted: June 18, 2020 Accepted: March 25, 2021 Published: May 10, 2021

Reference information: /CI Insight. 2021;6(9):e141426. https://doi.org/10.1172/jici. insight.141426.

\section{Introduction}

Achondroplasia ( $\mathrm{ACH})$, the most common form of dwarfism, is due to a gain-of-function mutation in the fibroblast growth factor receptor type 3 (FGFR3) gene $(1,2)$. FGFR3 is expressed in growth plate cartilage and bone, which explains the bone anomalies observed in patients with $\mathrm{ACH}$. The characteristic features of these patients are short arms and legs, macrocephaly, hypoplasia of the midface, lordosis, foramen magnum stenosis, and spinal compression (3). The generation of Fgfr3-specific mouse models has highlighted the role of FGFR3 during bone growth. In the absence of Fgfr3, the most prominent phenotype of the mice is overgrowth, thus indicating that FGFR3 is a negative regulator of bone growth $(4,5)$. Conversely, mice expressing a Fgfr3-activating mutation develop dwarfism and have reduced linear growth and impaired endochondral ossification, with reduced chondrocyte proliferation and reduced hypertrophic differentiation (6-10). A complex intracellular network of signals, including FGFR3, mediates this skeletal phenotype. Activating mutations in FGFR3 lead to upregulated FGFR3 protein (11) and to increased activity of several downstream intracellular signaling pathways, including MAPK, PI3K/AKT, PLC $\gamma$, and STATs (12).

During development, the rate of longitudinal bone growth is determined by chondrocyte proliferation and differentiation and is regulated by several secreted growth factors and endocrine factors, including parathyroid hormone-like peptide, Indian Hedgehog, bone morphometric proteins, transforming growth factor $\beta$, insulin like growth factor, and C-type natriuretic peptide (CNP, ref. 13). CNP and its receptor, the guanylyl cyclase natriuretic peptide receptor 2 (NPR2, also known as guanylyl cyclase B), are expressed in chondrocytes as well as in osteoblasts and are recognized as important regulators of longitudinal bone growth and bone homeostasis. NPR2 possesses guanylyl cyclase activity that leads to synthesis of cyclic guanosine monophosphate (cGMP), and dysregulation of this pathway is responsible for skeletal disorders. In clinical studies, inactivating mutations of NPR2 were found to cause a rare form of extreme short stature, called acromesomelic dysplasia, 
type Maroteaux (14-16). Conversely, heterozygous NPR2 gain-of-function mutations cause tall stature (17), and overexpression of CNP due to a balanced translocation is responsible for overgrowth and bone anomalies $(18,19)$. Mouse models with Npr2 loss-of-function mutations or with disruption of the CNP gene (Nppc) also show severe dwarfism (20-24), and an Npr2 gain-of-function mutation causes overgrowth (25). All of these data support a key role of the CNP/NPR2 signaling pathway for normal growth.

Previous studies have indicated that, among its diverse signaling effects, activation of FGFR3 results in reduced phosphorylation and activity of NPR2 in the growth plate $(26,27)$. Because CNP activation of NPR2 requires that the receptor is phosphorylated on multiple serines and threonines $(28,29)$, FGF-induced NPR2 dephosphorylation lowers cGMP and opposes bone growth. The significance of this aspect of FGF signaling for $\mathrm{ACH}$ was definitively established by the recent finding that, in a mouse model of $\mathrm{ACH}$, bone growth is restored by replacing the NPR2 protein with a dephosphorylation resistant form of NPR2 (NPR2 ${ }^{\text {TE/7E }}$, also known as GC-B ${ }^{7 \mathrm{E} / 7 \mathrm{E}}$ ) with a modified version of the protein that cannot be dephosphorylated (30). Treatment with CNP or a protease-resistant CNP analog, known as BMN-111 or vosoritide, also increases bone growth in mouse models of $\mathrm{ACH}(31,32)$, and $\mathrm{BMN}-111$ is currently in clinical development, with phase 2 and 3 results showing additional height gain in $\mathrm{ACH}$ patients $(33,34)$. These accumulating results, together with evidence that a PPP-family phosphatase mediates the FGF-induced dephosphorylation and inactivation of NPR2 $(26,27)$, suggest that a PPP-family phosphatase inhibitor could enhance bone growth in ACH patients if applied together with a CNP analog.

Here, we tested this concept using a semiselective PPP family phosphatase inhibitor, LB-100 (35). In studies of animal cancers, LB-100 has been shown to enhance responses to immunotherapy, CAR T cell therapy, and tyrosine kinase inhibitors (36-38). Phase 1 clinical trials concluded that the safety, tolerability and preliminary evidence of antitumor activity supported continued testing as a potentially novel treatment for human cancers (39). Here, we find that LB-100 counteracts the FGF-induced dephosphorylation and inactivation of NPR2, complementing the CNP stimulation and promoting bone growth in a mouse model of $\mathrm{ACH}$. Our results provide evidence for the concept that an inhibitor of NPR2 dephosphorylation could be used together with an NPR2 agonist to enhance cGMP production as a therapy for ACH.

\section{Results}

LB-100 counteracts the inactivation of Npr2 by FGF in growth plate chondrocytes. NPR2 activity in chondrocytes of intact growth plates was measured as previously described, using mice expressing a FRET sensor for cGMP, cGi500 (27). The mice were WT for Fgfr3. Tibias were isolated from newborn mice, and the overlying tissue was excised to expose the growth plate for confocal imaging (Figure 1A). When the NPR2 agonist CNP was perfused across the growth plate, the CFP/YFP emission ratio from cGi500 increased, indicating an increase in cGMP, due to stimulation of the guanylyl cyclase activity of NPR2 (Figure 1B). Similar results were obtained with protease-resistant BMN-111 (Supplemental Figure 1; supplemental material available online with this article; https://doi.org/10.1172/jci.insight.141426DS1). Perfusion of A-type natriuretic peptide (ANP), which activates the NPR1 guanylyl cyclase, or perfusion of a nitric oxide donor (DEA/NO), which activates soluble guanylyl cyclases, did not increase cGMP (Supplemental Figure 2), showing that — among the several mammalian guanylyl cyclases - only NPR2 is active in the chondrocytes of the mouse growth plate. As previously shown (27), exposure of the growth plate to FGF18 suppressed the cGMP increase in response to CNP perfusion (Figure 1B), indicating that FGF receptor activation decreases NPR2 activity.

Based on previous evidence that a PPP-family phosphatase inhibitor, cantharidin $(100 \mu \mathrm{M})$, inhibits the inactivation of NPR2 in growth plate chondrocytes by FGF (27), we tested whether a less toxic cantharidin derivative, LB-100, would increase NPR2 activity and long bone growth. LB-100 was originally reported as a specific inhibitor of the catalytic subunit of PPP2 (PPP2C) but was later shown to also act as a catalytic inhibitor of PPP5C (35). Since cantharidin demonstrates only modest selectivity for PPP2C versus PPP1C (40), we tested the ability of LB-100 to inhibit PPP1C activity using 2 established assays that use different substrates. We determined that LB-100 also inhibits PPP1C with an $\mathrm{IC}_{50}<2 \mu \mathrm{M}$ (Figure 1C and Table 1). Based on its structural similarity with cantharidin, $10 \mu \mathrm{M} \mathrm{LB}-100$ is not likely to inhibit PPP3C/calcineurin or PPP7C/PPPEF (41).

To investigate if LB-100 counteracts the inactivation of NPR2 by FGF, we preincubated the tibia with or without LB-100 and then with FGF. Following these incubations, the tibia was placed in a perfusion slide for confocal imaging, and cGMP production by NPR2 was monitored by measuring the increase in the CFP/ YFP emission ratio in response to CNP. The 2-hour incubation with $10 \mu \mathrm{M}$ LB-100 caused no visible change in chondrocyte morphology, as imaged in the live growth plate (compare Figure $1 \mathrm{D}$ with the control in Figure 1A). 
A

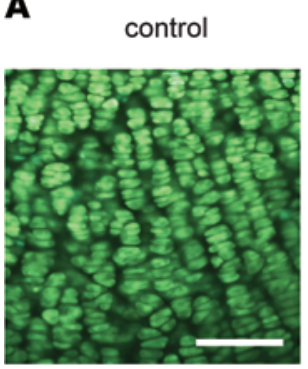

D

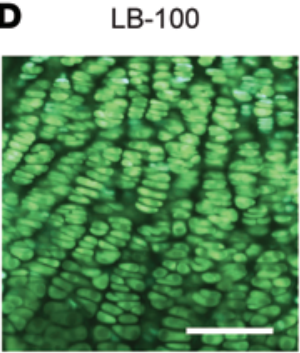

B

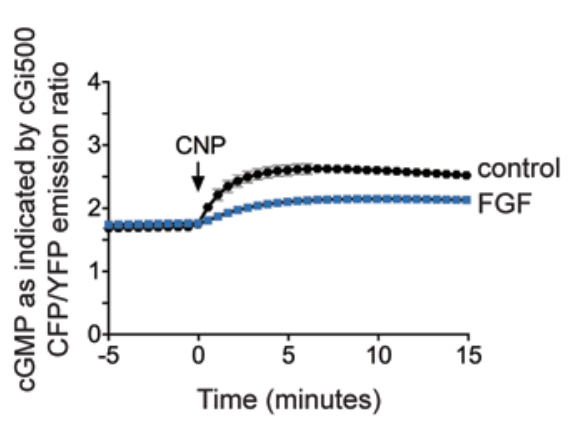

E

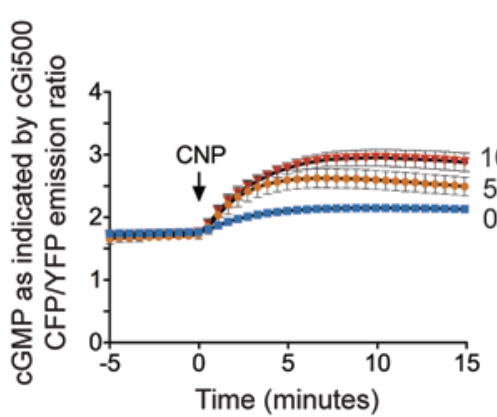

C

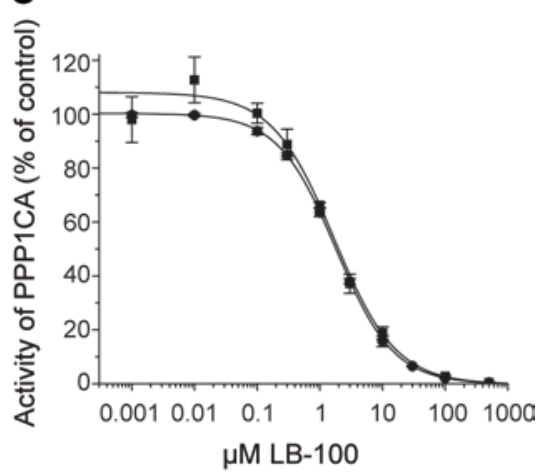

$\mathbf{F}$

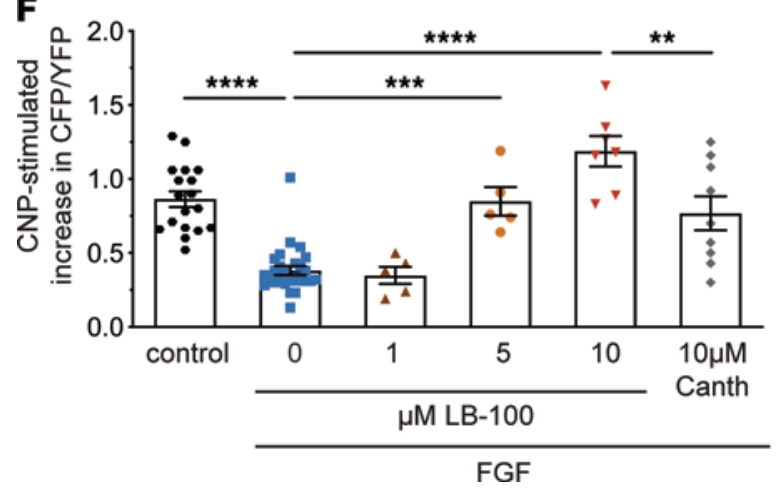

Figure 1. LB-100 counteracts the inactivation of NPR2 by FGF in growth plate chondrocytes of intact tibias from newborn mice. (A) Confocal image of cGi500 fluorescence in chondrocytes in the columnar and prehypertrophic region of the tibia growth plate, as used for measurements of cGMP production. Scale bar: $100 \mu \mathrm{m}$. (B) cGMP increase in growth plate chondrocytes in response to perfusion of $0.1 \mu \mathrm{M}$ CNP, and inhibition of the cGMP increase by pretreatment with $0.5 \mu \mathrm{g} / \mathrm{mL}$ FGF1 $8+1 \mu \mathrm{g} / \mathrm{mL}$ heparin for 80 minutes. Control tibias were pretreated for 80 minutes with heparin only. The graph shows the mean \pm SEM for 27 control tibias and 18 FGF-treated tibias. (C) Inhibitory effect of LB-100 on the activity of recombinant PPP1CA by LB-100 assayed using DiFMUP (diamonds) or [ $\left.{ }^{32} \mathrm{P}\right]$-labeled histone (squares) as a substrate. Each point represents the mean $\pm \mathrm{SD}(n=3-4)$. IC ${ }_{50}$ values are provided in Table 1 . (D) Confocal image of $\mathrm{cGi500}$ fluorescence in growth plate chondrocytes after pretreatment with $10 \mu \mathrm{M}$ LB-100 for 2 hours. No difference in morphology was seen compared with control tibias (A) without LB-100. Scale bar: $100 \mu \mathrm{m}$. (E and F) Effect of LB-100 (or cantharidin) preincubation on CNP-stimulated cGMP production in FGF-treated tibias. Tibias expressing cGi500 were preincubated with solutions with or without LB-100 for 60 minutes. FGF was then added, and 80 minutes later, tibias were placed in a perfusion slide for cGi500 imaging during CNP perfusion. (E) The CFP/YFP emission ratio as a function of time after CNP perfusion. (F) The CFP/YFP emission ratio at 15 minutes after CNP perfusion. Symbols indicate individual tibias $(n=5-27)$. For $\mathbf{E}$ and $\mathbf{F}$, data are shown as mean \pm SEM. Data were analyzed by 1-way ANOVA followed by the Holm-Sidak multiple comparison test. ${ }^{* *} P<0.01,{ }^{* * *} P<0.001,{ }^{* * * *} P<0.0001$.

After FGF treatment, the cGMP increase in response to CNP was small (Figure 1E). However, when the tibia was preincubated with 5 or $10 \mu \mathrm{M}$ LB-100 before applying FGF, the CNP-induced cGMP increase was enhanced (Figure 1, E and F). A concentration of $1 \mu \mathrm{M}$ LB-100 had no effect (Figure 1F). The CFP/YFP emission ratio attained after CNP perfusion in tibias that had been incubated in 5 or $10 \mu \mathrm{M}$ LB-100 before the FGF treatment was similar to or greater than the ratio in control tibias without FGF (Figure 1F). Figure 1F summarizes the CNP-stimulated increases in the CFP/YFP emission ratio from cGi500 under these various conditions and demonstrates that LB-100 counteracts the inactivation of NPR2 by FGF. LB-100 was more effective than cantharidin, with $5 \mu \mathrm{M}$ LB-100 resulting in a stimulation equivalent to that seen with $10 \mu \mathrm{M}$ cantharidin (Figure 1F). 
Table 1. Inhibitory activity of LB-100 against PPP1C, PPP2C, and PPP5CA.

\begin{tabular}{|c|c|c|c|}
\hline \multirow[t]{2}{*}{ Substrate } & \multicolumn{3}{|c|}{$\begin{array}{c}\text { Phosphatase } \\
\mathrm{IC}_{50} \text { mean } \pm \mathrm{SE}(\mu \mathrm{M})^{\mathrm{B}}\end{array}$} \\
\hline & PPP1C & PPP2C & PPP5C \\
\hline DiFMUP & $1.80 \pm 0.021$ & $0.39 \pm 0.013$ & $1.82 \pm 0.093$ \\
\hline $\begin{array}{c}{\left[{ }^{32} \mathrm{P}\right] \text {-labeled }} \\
\text { phosphohistone }\end{array}$ & $1.68 \pm 0.13$ & $0.64 \pm 0.037$ & $4.9 \pm 0.29$ \\
\hline
\end{tabular}

${ }^{A}$ Phosphatase activity was measured as described in the Methods. ${ }^{\mathrm{I}} \mathrm{C}_{50}$ values were calculated from a 10 -point concentration/dose response curve by a 4-parameter logistic fit of the data, using 3-8 replicates per concentration. 'Values for PPP2C and PPP5C have been reported previously (35). PPP1C, PPP2C, and PPP5C assays were conducted using the same assays, conducted by the same investigators, and employed the same batch of substrate and assay conditions.

LB-100 counteracts the FGF-induced dephosphorylation of NPR2 by FGF in primary chondrocyte cultures. To investigate if LB-100 counteracts the FGF-induced dephosphorylation of NPR2, we used Phos-tag gel electrophoresis (42) to analyze the phosphorylation state of NPR2 in isolated chondrocytes from the ribs of newborn mice. The mice were WT for Fgfr3. To allow specific labeling of the NPR2 protein, the mice were genetically modified to insert a 9-amino acid hemagglutinin (HA) tag on the N-terminus of NPR2 (HA-Npr2; ref. 43) (Supplemental Figure 3). We compared the phosphorylation state of NPR2 in chondrocytes with and without LB-100 preincubation - and with and without subsequent exposure to FGF. Treated and untreated chondrocytes had a similar appearance (Supplemental Figure 4).

Chondrocyte proteins were separated by Phos-tag gel electrophoresis, which slows migration of phosphorylated proteins, and Western blots were probed for NPR2 (Figure 2A). Without FGF treatment, NPR2 protein from the rib chondrocytes was present in a broad region of the gel. With FGF treatment, the ratio of the signal in the upper versus lower regions decreased (Figure 2, A and B; compare lanes 1 and 2), indicating NPR2 dephosphorylation in response to FGF and confirming, with primary chondrocytes, a previous study using a rat chondrosarcoma (RCS) cell line (26). However, if the chondrocytes were preincubated with $10 \mu \mathrm{M}$ LB-100, the dephosphorylation in response to FGF was only partial, indicating that LB-100 counteracts the FGF-induced dephosphorylation of NPR2 (Figure 2, A and B; compare lanes 2 and 4).

To more closely mimic conditions used in experiments to be described below, the NPR2 phosphorylation state was also analyzed using chondrocyte cultures to which we added the protease-resistant BMN111. The addition of BMN-111 caused some reduction in NPR2 phosphorylation (Figure 2, A and B; compare lanes 1 and 5), independently of treatment with FGF (Figure 2, A and B; compare lanes 2 and 6). This is consistent with previous evidence that some NPR2 dephosphorylation occurs in response to prolonged agonist (CNP) exposure (28). However, the addition of BMN-111 did not change the conclusions that FGF causes NPR2 dephosphorylation (Figure 2, A and B; compare lanes 5 and 6) and that LB-100 counteracts the FGF-induced dephosphorylation (Figure 2, A and B; compare lanes 6 and 8).

In Fgfr $3^{\mathrm{Y367C/+}}$ femurs, LB-100 enhances the stimulation of bone growth by the protease-resistant NPR 2 agonist $B M N$-111. Previously, we showed that the protease-resistant CNP analog BMN-111 increases bone growth in a mouse model of $\mathrm{ACH}$, in which tyrosine 367 is changed to a cysteine $\left(\mathrm{Fg} f 3^{\mathrm{Y3} 67 \mathrm{C} / \mathrm{+}}\right)$, resulting in constitutive activation of FGFR3 $(32,44)$. However, BMN-111 only partially rescued the effect of the FGFR3-activating mutation. Our finding that LB-100 opposes the FGF-induced dephosphorylation and inhibition of NPR2 activity in chondrocytes suggested that applying LB-100 together with BMN-111 might enhance the stimulation of growth in bones from $\mathrm{Fg} f \mathrm{r}^{\mathrm{Y3} 36 \mathrm{Cl} /+}$ mice (Figure 3A).

As previously reported (32), $0.1 \mu \mathrm{M}$ BMN-111 increased the growth of cultured femurs from E16.5 Fgfr $3^{\mathrm{Y3} 67 \mathrm{Cl}+\mathrm{+}}$ mice (Figure 3, B-D). Over a 6-day culture period, the mean increase in bone length in the BMN111-stimulated Fgfr $3^{\mathrm{Y3} 67 \mathrm{C} / \mathrm{+}}$ femurs was 1.78 times that in vehicle-treated bones (Figure 3C). LB-100 alone also increased the extent of elongation, showing a growth ratio of 1.30 for LB-100/control (Figure 3C). However, when $\mathrm{Fg} f \mathrm{r}^{\mathrm{Y3} 67 \mathrm{C} /+}$ femurs were cultured with BMN-111 together with $10 \mu \mathrm{M}$ LB-100, the mean increase in bone length was 2.06 times that in untreated bones (Figure 3C). Thus, the combination of BMN-111 and LB-100 resulted in elongation during the culture period that was 16\% greater than with BMN-111 alone.

We also measured the effect of LB-100 and BMN-111 on the increase of the total bone and cartilage area, defined as the area within the periphery of a photograph of the femur (Supplemental Figure 5). LB-100 and 

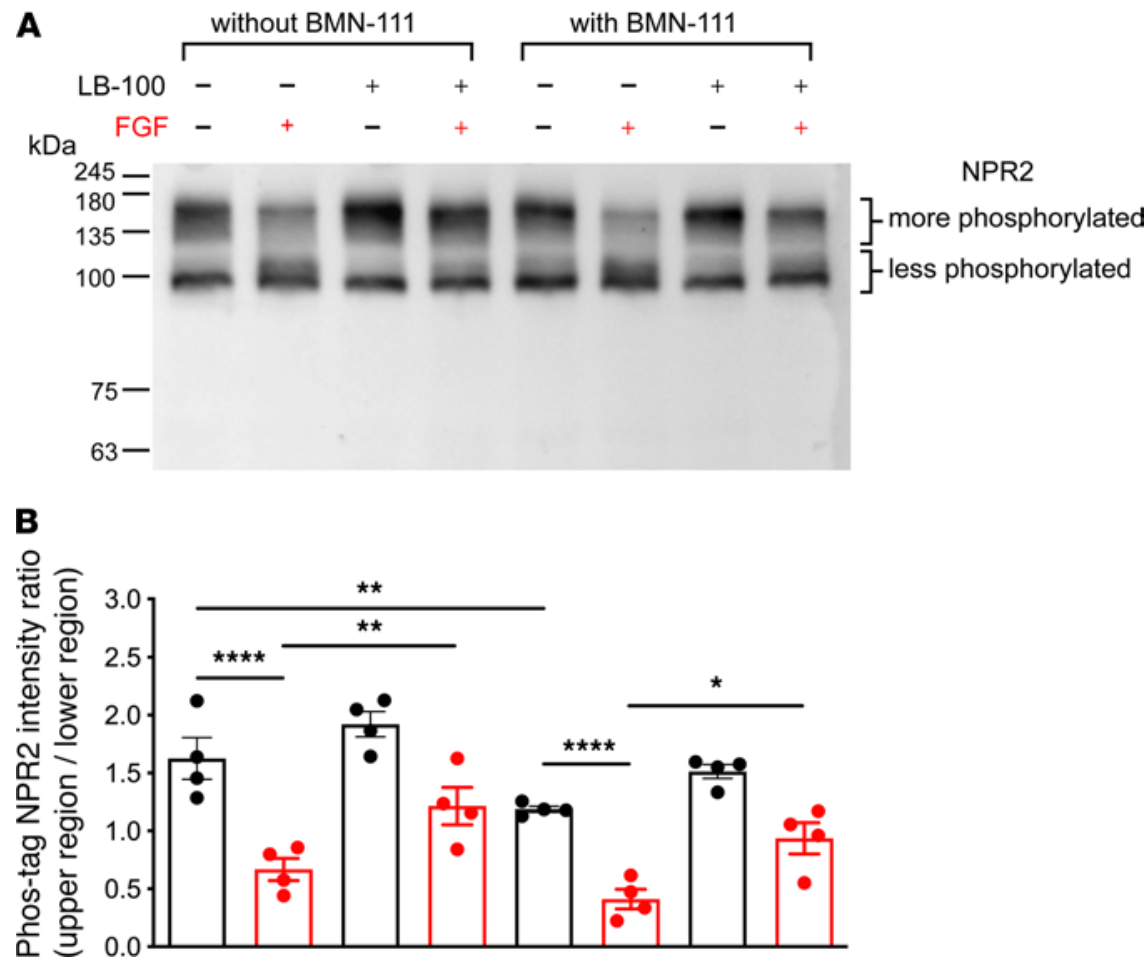

Figure 2. LB-100 counteracts the FGF-induced dephosphorylation of NPR2 in primary chondrocyte cultures. (A) Western blot of a Phos-tag gel showing the decrease in NPR2 phosphorylation in chondrocytes treated with FGF18 $(0.5 \mu \mathrm{g} / \mathrm{mL})$ for 10 minutes and attenuation of this dephosphorylation in chondrocytes pretreated with LB-100 $(10 \mu \mathrm{M})$ for 1-hour prior to treatment with FGF18 (lanes 1-4). Lanes 5-8 show samples that were treated similarly, except that the protease-resistant CNP analog BMN-111 $(0.1 \mu \mathrm{M})$ was present during the 1-hour pretreatment period. Chondrocytes were obtained from mice in which the endogenous NPR2 was tagged with an HA epitope. Blots were probed with an antibody recognizing the HA epitope. (B) Densitometry measurements for 4 experiments like that shown in $\mathbf{A}$. The $y$ axis indicates the ratio of the intensity of the upper region to that of the lower region as shown in $\mathbf{A}$; a smaller ratio indicates a decrease in NPR2 phosphorylation. Symbols indicate individual experiments; data are shown as mean \pm SEM. Data were analyzed by repeated measures 2-way ANOVA followed by Holm-Sidak multiple comparisons tests between the indicated groups. ${ }^{*} P<0.05 ;{ }^{* *} P<0.01 ;{ }^{* * * *} P<0.0001$.

BMN-111 each individually increased this area, with a growth ratio of 1.40 for LB-100/control, and a growth ratio of 1.51 for BMN-111/control (Figure 3D). The combination of LB-100 and BMN-111 was even more effective, with a growth ratio of 1.93 . Thus, the combination of BMN-111 and LB-100 enhanced the increase in bone and cartilage area by $27 \%$ compared with BMN-111 alone (Figure 3D).

Combined treatment with $\mathrm{LB}-100$ and BMN-111 improves growth plate cartilage homeostasis in $\mathrm{Fgfr} 3^{\mathrm{Y3} 67 \mathrm{C} /+}$ femurs. Histological analyses of the epiphyseal growth plates of $F g f r 3^{Y 367 C /+}$ femurs showed that combining BMN-111 and LB-100 treatments improved cartilage growth homeostasis (Figure 4). Prehypertrophic and hypertrophic chondrocytes produce an extracellular matrix rich in Collagen type X (COLX); we used COLX immunostaining to label the hypertrophic region and to visualize and measure individual cells. This labeling revealed a highly beneficial effect of the combined treatment on the size of the cells in the hypertrophic area of $\mathrm{Fg} f \mathrm{r}^{\mathrm{Y367C/+}}$ femurs (Figure 4, A and B). The mean cross-sectional area of individual hypertrophic chondrocytes of $\mathrm{Fg} f \mathrm{r}^{\mathrm{Y367C/+}}$ mice was reduced by about half compared with that in the $\mathrm{Fgfr}^{+/+}$growth plate (Figure 4, A and B; measured as described in Supplemental Figure 6). As previously reported (32), BMN-111 increased the size of the $F g f r 3^{Y 367 C /+}$ hypertrophic chondrocytes, but the cells remained smaller than for the WT (Figure 4, A and B). However, with the combined treatment of BMN-111 and LB-100, the mean area of the $\mathrm{Fg} f \mathrm{r}^{\mathrm{Y3} 67 \mathrm{C} /+}$ hypertrophic cells in the proximal growth plate was $32 \%$ greater than with BMN-111 alone and was similar to that of $\mathrm{Fgfr}^{+/+}$hypertrophic cells, indicating that the final differentiation of the chondrocytes was restored by the treatment (Figure 4, A and B). Corresponding measurements for the distal growth plate showed a similar trend (Supplemental Figure 7).

We also observed a beneficial effect of the combined treatment on the proliferative region of the growth plate of $\mathrm{Fg} f \mathrm{r}^{\mathrm{Y367C/+}}$ mice. We measured the area of the proliferative region by subtracting the hypertrophic 
A FGFR3
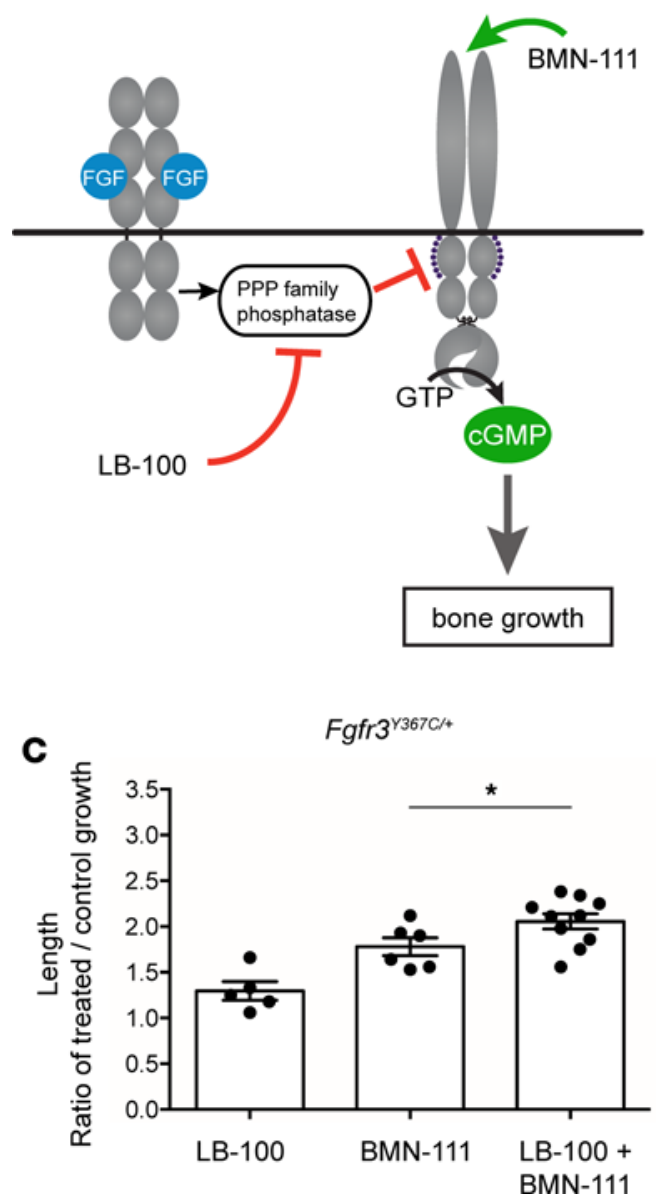

B

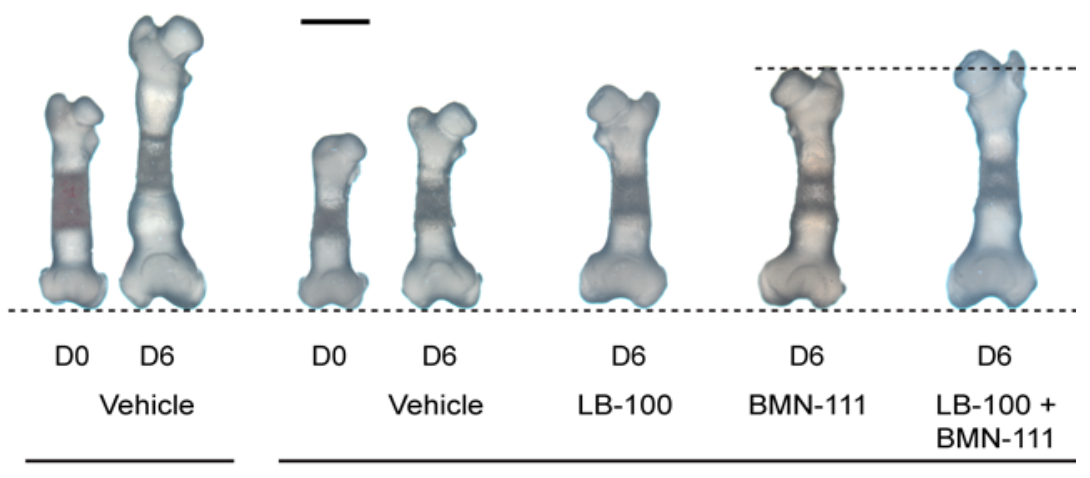

Fgfr $^{+/+}$

$\mathrm{Fgfr}^{\mathrm{Y} 367 \mathrm{C} / \mathrm{+}}$

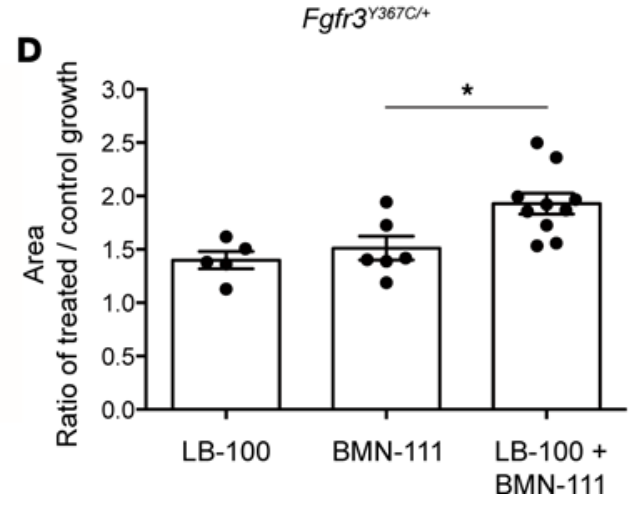

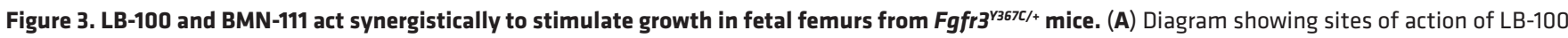
and BMN-111. (B) Representative photographs of fetal femurs from E16.5 Fgfr3 ${ }^{+/+}$(WT) and $\mathrm{Fgfr}^{\mathrm{Y} 367 \mathrm{C} /+}$ mice, before (D0) and after a 6-day (D6) culture with the indicated treatments. The upper dashed line indicates the groups compared in $\mathbf{C}$ and $\mathbf{D}$. Scale bar (solid line): $1 \mathrm{~mm}$. (C and $\mathbf{D})$ Measurements of growth in bone length (C) and area (D), showing that LB-100 + BMN-111 increases growth more than BMN-111 alone. The concentration of LB-100 was $10 \mu M$, and the concentration of BMN-111 was $0.1 \mu \mathrm{M}$. Symbols in graphs $\mathbf{C}$ and $\mathbf{D}$ indicate individual bones $(n=5-10)$. Data are shown as mean \pm SEM, and data were analyzed by unpaired 2 -tailed $t$ tests. ${ }^{*} P<0.05$ between indicated groups.

area, identified by COLX labeling, from the total growth plate area. Based on these measurements, the combined treatment increased the total proliferative growth plate area of the femur by an average of $33 \%$ over vehicle, compared with $20 \%$ for BMN-111 alone (Figure 4C). Thus, the combined treatment increased the proliferative area by $13 \%$ compared with BMN-111 alone (Figure 4C).

CNP signaling through NPR2 in the growth plate inhibits the MAP kinase pathway and its extracellular signal-regulated kinase 1 and 2 (ERK1/2) (refs. 31, 45, 46; Figure 5A). Therefore, we investigated the impact of treatment with LB-100 and BMN-111 on the phosphorylation of ERK1/2 in growth plates from $\mathrm{Fgfr}^{\mathrm{Y} 36 \mathrm{CC} /+}$ embryos. As expected, immunolabeling showed a high level of phosphorylated ERK1/2 in the proximal and distal parts of the cartilage compared with WT controls (Figure 5, B and C). The combined LB-100 and BMN-111 treatment of $\mathrm{Fgfr}^{3} \mathrm{Y36CC}^{\mathrm{Y} /+}$ femurs decreased the activity of the MAP kinase pathway, as demonstrated by the decreased phosphorylation of ERK1/2 in the proximal and distal growth plates of the femurs (Figure 5, B and C).

The combination of LB-100 and BMN-111 enhances growth and improves chondrocyte differentiation in the ex vivocultured Fgfr3 $3^{Y 36 \mathrm{CC} /+}$ skull base. Because compression of the spinal cord at the level of the foramen magnum (part of the skull base) is a critical clinical feature of $\mathrm{ACH}$, contributing significantly to infant morbidity, we tested whether the combination of LB-100 and BMN-111 improves the defective growth of the skull base observed in $\mathrm{Fgfr}^{\mathrm{Y} 36 \mathrm{CC} / \mathrm{+}}$ mice. To investigate this question, we developed a model of ex vivo culture of the skull base isolated from mouse embryos (E16.5; Figure 6A). Over a 6-day culture period, we observed that the elongation of the 
A
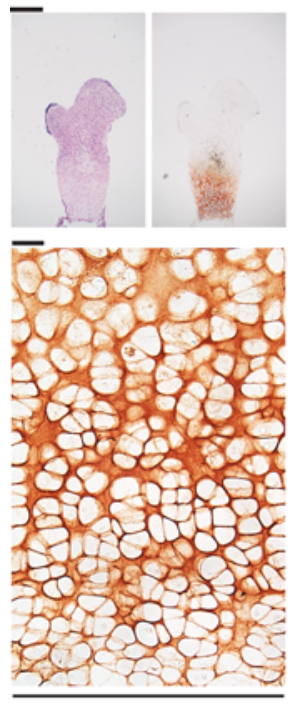

Vehicle
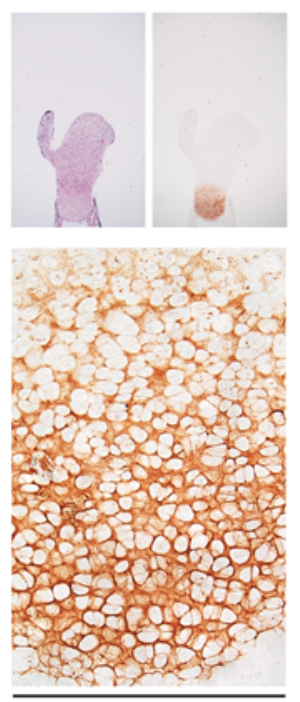

Vehicle
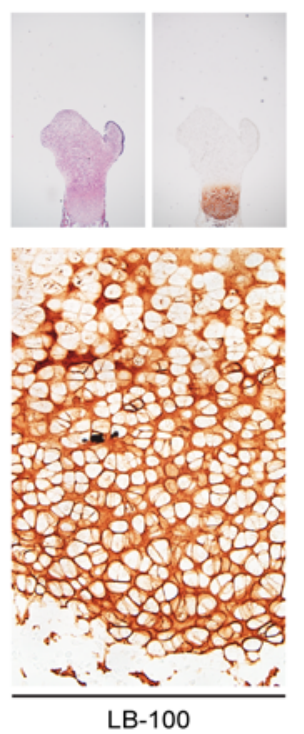
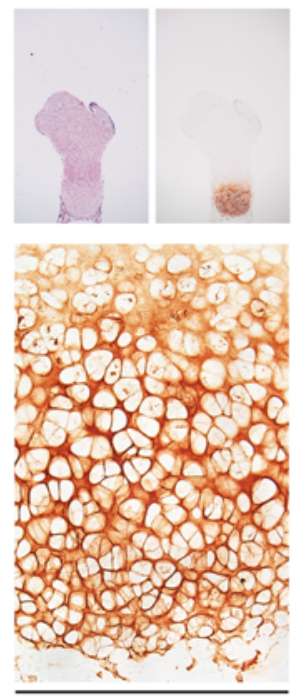

BMN-111
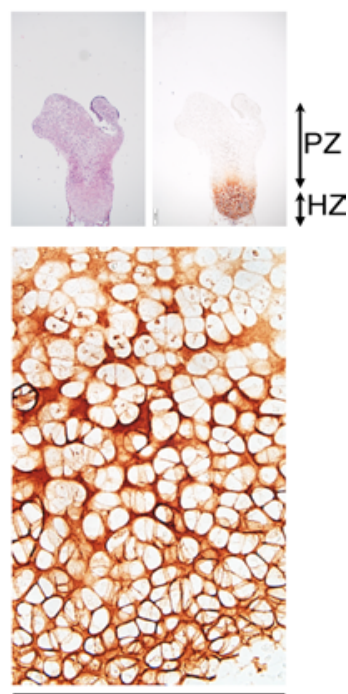

LB-100 + BMN-111
$\mathrm{Fgfr}^{+/+}$

B

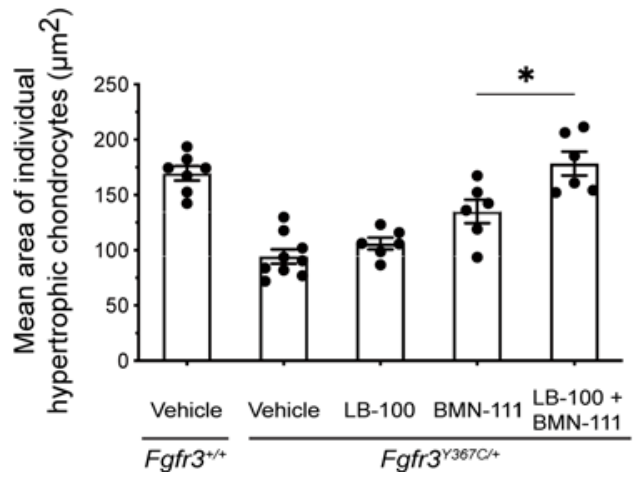

$\mathrm{Fgfr}^{\mathrm{r} 367 \mathrm{C} / \mathrm{t}}$

c

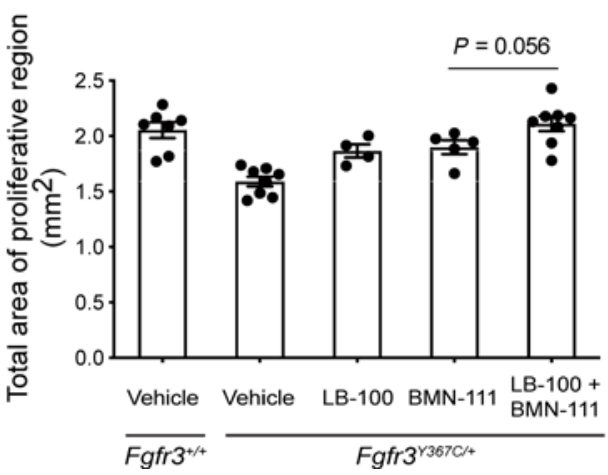

Figure 4. Dual action of LB-100 and BMN-111 improves chondrocyte differentiation and increases the proliferative growth plate area of ex vivo cultured

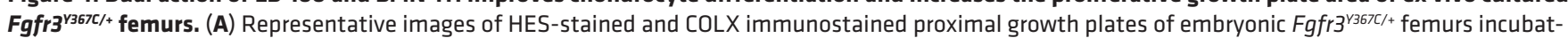
ed for 6 days with vehicle or LB-100 $(10 \mu \mathrm{M})$ and/or BMN-111 $(0.1 \mu \mathrm{M})$. Growth plates of $F g f \mathrm{~F}^{+/+}$femurs cultured with vehicle are also shown. PZ, proliferative zone; $\mathrm{HZ}$, hypertrophic zone. Scale bars: $400 \mu \mathrm{m}$ (upper row), $20 \mu \mathrm{m}$ (lower row). (B) Mean area of individual hypertrophic chondrocytes in proximal growth plates of femurs treated as described for $\mathbf{A}$ ( $n=6-9$ bones measured for each condition, with 54-185 cells measured for each bone). (C) Total proliferative area of the proximal plus distal growth plates of femurs treated as described for $\mathbf{A}(n=4-8$ bones measured for each condition). Symbols represent individual bones. Data are shown as mean \pm SEM. Data were analyzed by 2 -tailed unpaired $t$ tests between the indicated groups. ${ }^{*} P<0.05$.

cranial base was altered in explants from $\mathrm{Fgfr}^{\mathrm{Y3} 36 \mathrm{Cl} /+}$ embryos compared with $\mathrm{Fgfr}^{3^{+/+}}$embryos, because of a reduced size of the spheno-occipital and interoccipital synchondroses, localized respectively between the basioccipital bone (BO) and basisphenoid bone (BS), and between the interoccipital bone (IO) and BO (Figure 6, B-D).

The combination of LB-100 and BMN-111 increased the percent of growth of the 2 synchondroses in explants from $\mathrm{Fgfr} \mathrm{YY}^{\mathrm{Y} 36 \mathrm{CC} /+}$ embryos (Figure 6, C and D), leading to a rescue of the skull base anomalies, with similar bone elongation comparing $\mathrm{Fgfr}^{+/++}$and treated $\mathrm{Fg} f \mathrm{r}^{\mathrm{Y3} 36 \mathrm{Cl} /+}$ explants (Figure 6, C and D). Histological analyses of the synchondrosis showed that combined BMN-111 and LB-100 treatments improved cartilage homeostasis in $\mathrm{Fgfr}^{\mathrm{Y} 367 \mathrm{CC} / \mathrm{+}}$ explants (Figure 6E). COLX immunolabeling revealed a highly beneficial effect of the combined treatment on the size of the cells in the hypertrophic area of $\mathrm{Fgfr} 3 \mathrm{Y3}^{\mathrm{Y} 6 \mathrm{Cl} / \mathrm{C}}$ cartilage.

\section{Discussion}

Understanding of the mechanisms by which FGF/FGFR3 and CNP/NPR2 regulate longitudinal bone growth has allowed the development of an effective therapeutic strategy using a CNP analog (vosoritide; BMN-111) 
A
FGFR3

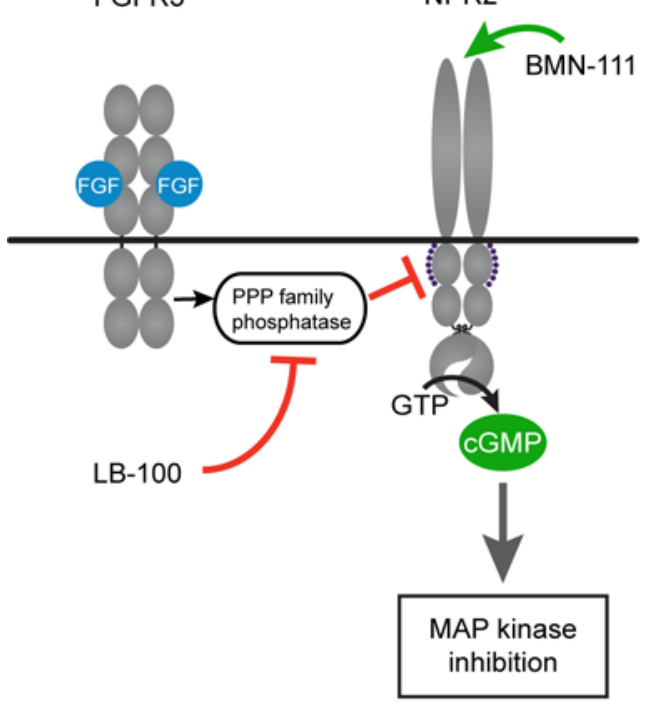

B
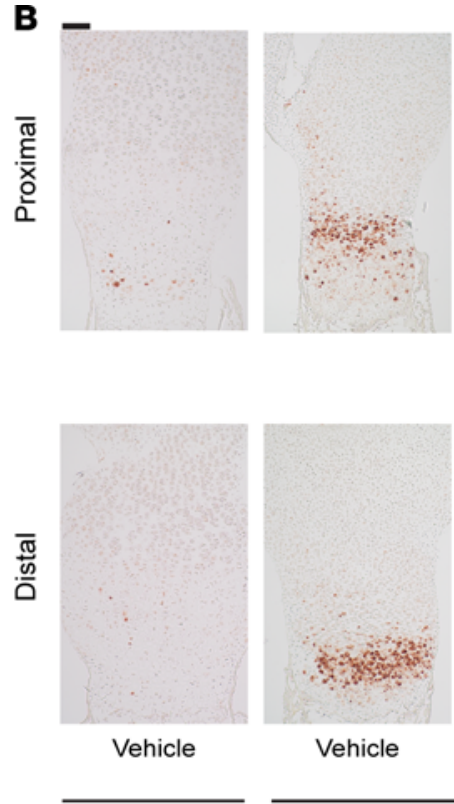

$\mathrm{Fgfr}^{+/ *}$

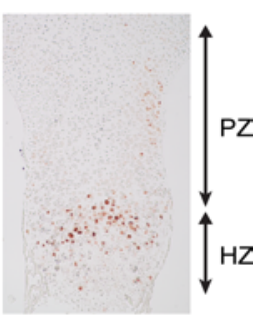

$\mathrm{PZ}$

$H Z$

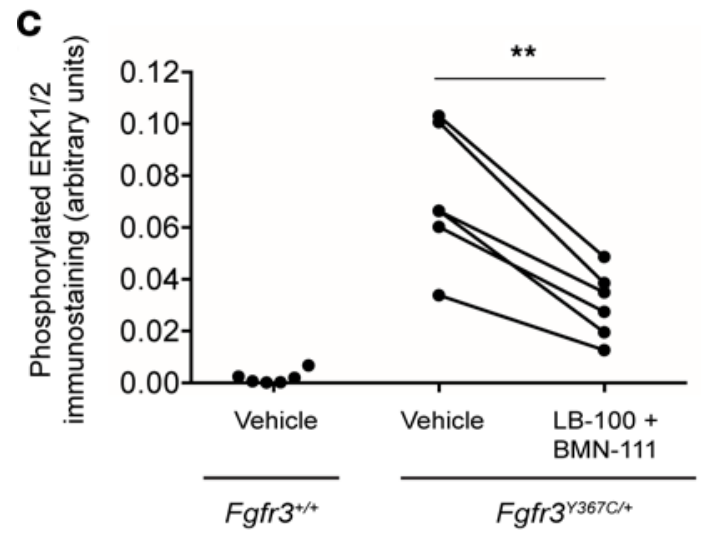

Figure 5. Dual action of LB-100 and BMN-111 decreases the activation of the MAP kinase pathway. (A) Diagram showing sites of action of LB-100 and BMN-111 on the MAP kinase pathway. (B) Representative images of phosphorylated ERK1/2 immunostaining of proximal and distal growth plates of embryonic $\mathrm{Fgfr}^{\mathrm{r} 36 \mathrm{Cl} / \mathrm{+}}$ femurs incubated for 6 days with vehicle or with LB-100 $(10 \mu \mathrm{M})$ and BMN-111 $(0.1 \mu \mathrm{M})$. Growth plates of WT $\left(\mathrm{Fgfr}^{+/+}\right)$femurs cultured with vehicle are also shown. Scale bar: $100 \mu \mathrm{m}$. (C) Quantitation of phosphorylated ERK1/2 immunostaining in proximal plus distal growth plates of femurs from the same $\mathrm{FgFr}{ }^{\mathrm{r} 36 \mathrm{C} / \mathrm{+}}$ animal treated with either vehicle or a combination of LB-100 and BMN-111. Symbols represent individual femurs ( $n=6$ bones per condition). Data were analyzed by paired $t$ test; ${ }^{* *} P<0.01$. Data from 6 vehicletreated $\mathrm{Fgfr}^{+/+}$femurs are shown for comparison.

to treat $\mathrm{ACH}(33,34)$. The findings described here identify the PPP-family phosphatase inhibitor LB-100 as a stimulator of bone growth when used in combination with this CNP analog to stimulate production of cGMP by NPR2. Firstly, using isolated WT bones incubated with FGF to mimic an ACH-like condition, we show that pretreatment with LB-100 counteracts the decrease in NPR2 guanylyl cyclase activity by FGFR3. Secondly, our results show that FGFR3 activation leads to NPR2 dephosphorylation in primary cultured WT chondrocytes and that LB-100 suppresses the dephosphorylation. Moreover, application of a combination of BMN-111 and LB-100 to long bones from the ACH mouse model $\mathrm{Fg} f \mathrm{r}^{\mathrm{Y} 336 \mathrm{CC} / \mathrm{+}}$ results in growth that exceeds that stimulated by BMN-111 alone, and this combination also increases growth of the cranial base. This beneficial impact of the treatment on skull base elongation in $\mathrm{Fg}_{\mathrm{gr}} 3^{\mathrm{Y3} 36 \mathrm{Cl} / \mathrm{+}}$ mice and the correction of their defects are promising because the stenosis of the foramen magnum of $\mathrm{ACH}$ patients results from defective cranial base elongation. These results provide a proof of concept that BMN-111 and a PPP-family phosphatase inhibitor could potentially be used in combination for treatment of skeletal dysplasias such as ACH.

Our data also show the benefit of this treatment for growth plate cartilage during bone development in Fofr ${ }^{3367 \mathrm{Cl} /+}$ mice. During the process of endochondral ossification, chondrocytes actively proliferate in the resting and proliferating chondrocyte zone and then differentiate to hypertrophic chondrocytes, which lose the capacity to proliferate. The terminally differentiated hypertrophic cells are removed by cell death or transdifferentiate into osteoblasts. It is well known that FGFR3 signaling decreases bone growth by inhibiting both proliferation and differentiation of chondrocytes (47), and it has been proposed that FGFR3 acts by way of ERK1/2 to restrict hypertrophic differentiation (48). Here, we showed that treatment with BMN-111 
A
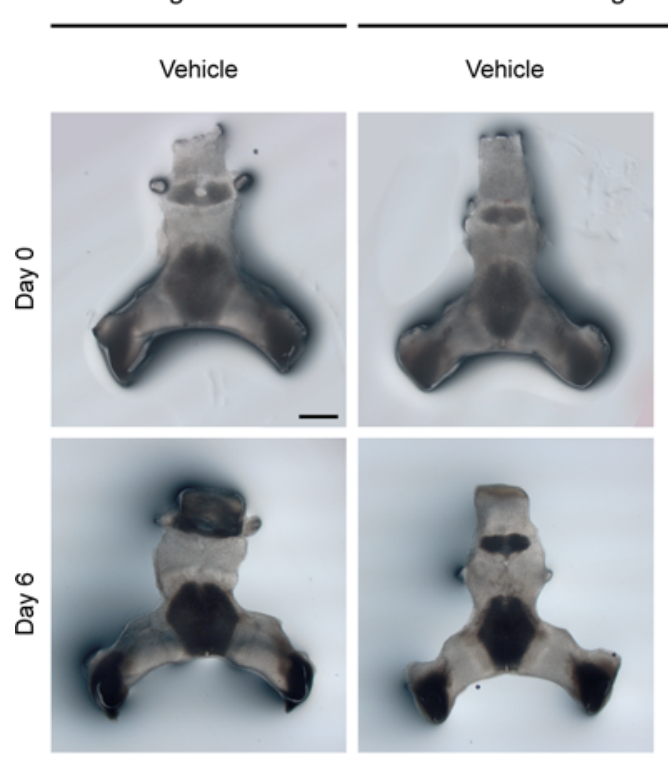

E
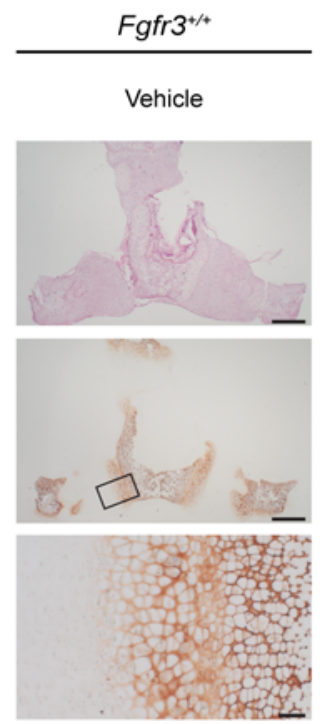

\section{LB-100 + BMN-111}
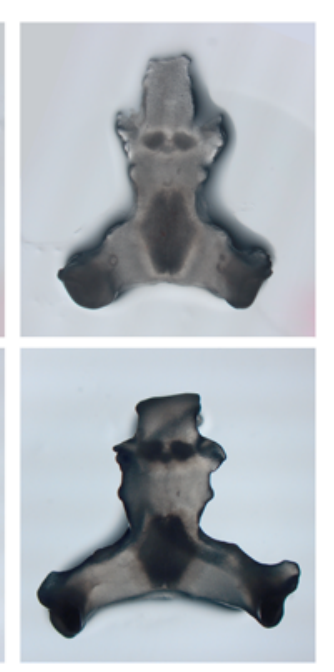

Fgfr $^{\text {r } 367 \mathrm{Cl} / 4}$

Fgfr $^{\text {r } 367 / /+}$
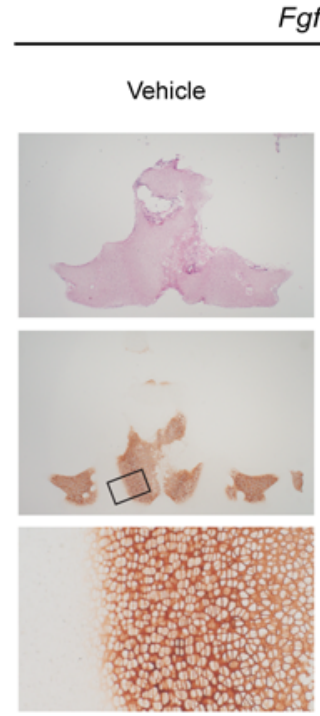

LB-100 + BMN-111
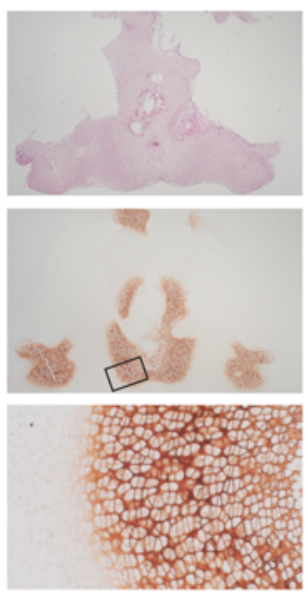

B
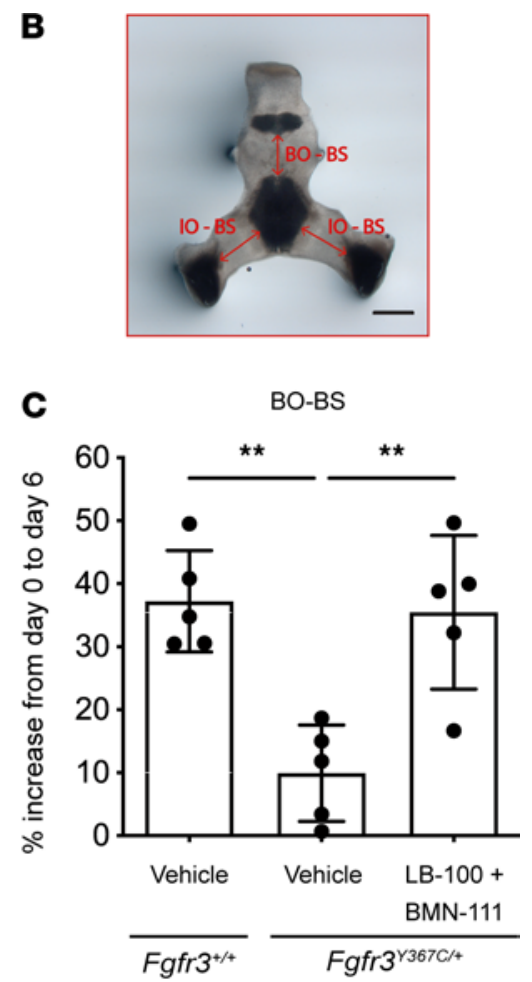

D

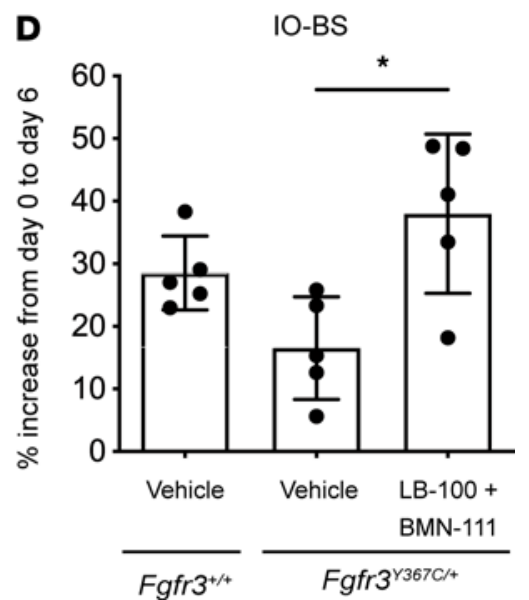

Figure 6. The combination of LB-100 and BMN-111 enhances growth and improves chondrocyte differentiation in the ex vivo cultured $\mathrm{Fgfr}^{\mathrm{r} 367 \mathrm{C} /+}$ skull base. (A) Photographs of ex vivo cultures of skull bases from $\mathrm{Fgfr}^{+/+}$and $\mathrm{Fgfr} \mathrm{F}^{\mathrm{Y} 36 \mathrm{CC} /+}$ embryos before (day 0 ) and after a 6-day culture with the indicated treatments. The concentration of LB-100 was $10 \mu \mathrm{M}$, and the concentration of BMN-111 was $0.1 \mu \mathrm{M}$. (B) Diagram of the measurement positions for the graphs shown in C and D. BO, basioccipital; BS, basisphenoid; IO, interoccipital. Scale bars: $500 \mu \mathrm{m}$ (A and B). (C and D) Percent increases in the indicated lengths between day 0 and day 6 . Symbols represent individual skull base explants $(n=5)$. Data are shown as mean \pm SEM. Data were analyzed by 1 -way ANOVA followed by Tukey's multiple comparison test. ${ }^{*} P<0.05$, ${ }^{* *} P<0.01$. (E) HES-stained and COLX immunostained embryonic Fgfr ${ }^{Y 3667 /+}$ skull bases treated for 6 days with vehicle or LB-100 $(10 \mu \mathrm{M})$ and BMN-111 $(0.1 \mu \mathrm{M})$. Skull bases of $\mathrm{Fgfr}^{+/+}$embryos cultured with vehicle are also shown. Representative images for each condition. Upper and middle rows, HES and COLX staining at low magnification; lower row, magnified view of COLX staining within the box marked in the middle row. Scale bars: $500 \mu \mathrm{m}$ (upper and middle rows), $50 \mu \mathrm{m}$ (lower row). Five samples for each condition.

and LB-100 reduced the levels of phosphorylated ERK1/2, thus modifying chondrocyte differentiation and allowing bone growth. In addition, we noted an impressive increase in the size of the hypertrophic cells. We concluded that the treatment restored cartilage homeostasis, and we hypothesize that the elevated cGMP resulting from this treatment could be a key regulator of transdifferentiation of hypertrophic cells into osteoblasts and could control the chondrogenic or osteogenic fate decision.

The increase in NPR2 phosphorylation by LB-100 is correlated with improved chondrocyte proliferation and differentiation in $\mathrm{Fgfr}^{\mathrm{Y3} 3 \mathrm{CC} /+}$ femurs, consistent with results with a mouse model $\left(\mathrm{Npr} 2^{7 \mathrm{E} / 7 \mathrm{E}}\right)$ mimicking 
constitutive phosphorylation of NPR2 $(27,30)$. Because LB-100 inhibits multiple PPP-family phosphatases (35) (Table 1), and because its safety for long-term use in children is unknown, our results provide only a proof of principle for a possible combination treatment. Future studies to determine which phosphatases act to dephosphorylate NPR2 in chondrocytes are clearly warranted, and increased height in children with mutations in particular PPP2 regulatory subunit genes provides a clue (49). Identification of these phosphatases and development of more specific inhibitors targeting them could lead to future therapies.

Recent mouse studies indicate that, in addition to increasing prepubertal bone elongation, phosphorylation of NPR2 increases bone density, due to an increase in the number of active osteoblasts at the bone surface (50). Because low bone density is one of the key clinical features of $\mathrm{ACH}$ (51), the combination a CNP analog and a phosphatase inhibitor could also have a beneficial impact on bone density for patients with $\mathrm{ACH}$ and related conditions. In addition, such a treatment could have potential for treatment of osteoporosis and, because CNP/NPR2 also plays a key role in regulation of joint homeostasis, could be beneficial for preventing or minimizing cartilage loss and promoting repair of the damaged articular cartilage in skeletal disorders and osteoarthritis (52). More generally, the combination of natriuretic peptides and phosphatase inhibitors could have therapeutic potential for multiple disorders involving NPR2 and the related guanylyl cyclase NPR1 that also requires phosphorylation for activity (53).

In summary, the combined (LB-100 and BMN-111) treatment acts on both chondrocyte proliferation and differentiation, thus promoting better bone growth. In $\mathrm{ACH}$, the homeostasis of the growth plate is disturbed, and proliferation and differentiation are affected by the overactivation of FGFR3. Currently, BMN-111 (vosoritide) is being studied in children with ACH and, as demonstrated in preclinical studies (32), mostly restores the defective differentiation in the growth plate. Recently reported phase 2 and 3 data demonstrate that BMN-111 results in a sustained increase in annualized growth velocity for up to 42 months in children 5-14 years of age with ACH ( 33 , 34). The present study provides a proof of concept that a combination of BMN-111 and a phosphatase inhibitor has the potential to increase bone growth rate in $\mathrm{ACH}$ patients to a higher level than BMN-111 alone.

\section{Methods}

Mice. Three mouse lines were used for this study: cGi500 (54), HA-Npr2 (43), and Fgfr3 ${ }^{\mathrm{Y} 36 \mathrm{C} /+}$ (44). The cGi500 mice were provided by Robert Feil (University of Tübingen, Tübingen, Germany). The strain for all mouse lines was CB7BL/6J; no sex selection was made. All experiments were performed using E16.5 embryos or 1- to 2-day-old newborns, as described for individual procedures.

Reagents. CNP and ANP were obtained from Phoenix Pharmaceutical (catalogs 012-03 and 005-24, respectively). BMN-111 was synthesized by New England Peptide as a custom order with the following sequence: (Cyc[23,39])H2N-PGQEHPNARKYKGANKKGLSKGCFGLKLDRIGSMSGLGC-OH, as previously described (32). The purity was $>95 \%$. DEA/NO was from Cayman Chemical (catalog 82100). LB-100 (3-[4-methylpiperazine-1-carbonyl]-7-oxabicyclo[2.2.1]heptane-2-carboxylic acid) was from Selleck Chemicals (catalog S7537) or MedChem Express (catalog HY-18597). Cantharidin was from Tocris (catalog 1548). FGF18 was from PeproTech (catalog 100-28), and heparin was from Sigma-Aldrich (catalog H4784). DiFMUP (6,8-Difluoro-4-methyl-7-[phosphonooxy]-2H-1-benzopyran-2-one) was from Thermo Fisher Scientific (catalog D6567).

Measurements of cGMP production in tibia growth plates using cGi500. cGMP production in chondrocytes within intact growth plates was measured using tibias dissected from newborn mice (0- to 1-day-old mice) that globally expressed 1 or 2 copies of the cGi500 FRET sensor, as previously described (27). Tibias were dissected and cultured overnight on Millicell organotypic membranes (PICMORG50; MilliporeSigma) in BGJb medium (Thermo Fisher Scientific, catalog 12591-038) with 0.1\% BSA (MP Biomedicals, catalog 103700), $100 \mathrm{units} / \mathrm{mL}$ of penicillin, and $100 \mu \mathrm{g} / \mathrm{mL}$ of streptomycin (Thermo Fisher Scientific, 15140 122). In preparation for imaging, each tibia was slit to remove the tissue overlying the growth plate. Where indicated, the tibia was incubated in LB-100, cantharidin, or control medium, followed by addition of FGF18 $(0.5 \mu \mathrm{g} / \mathrm{mL}+1 \mu \mathrm{g} / \mathrm{mL}$ heparin $)$ or control medium containing heparin only. The tibia was then placed in a perfusion slide (ibidi USA, catalog 80186, special order with no adhesive), and the growth plate was imaged on the stage of a confocal microscope, as previously described (27).

Determination of the effect of LB-100 on PPP1C phosphatase activity. The coding sequence of human PPP1CA was expressed as a maltose binding protein fusion in a BL-21 strain of $E$. coli and purified as previously described (55). Phosphohistone phosphatase assays were performed as previously described (55, 56). Briefly, LB-100, at the indicated concentrations, or vehicle control $\left(\mathrm{H}_{2} \mathrm{O}\right)$ was added to enzyme/buffer 
aliquots about 10 minutes prior to starting assays by the addition of $\left[{ }^{32} \mathrm{P}\right]$-phosphohistone substrate (to a final assay concentration of $300 \mathrm{nM}$ incorporated phosphate). $\left[{ }^{32} \mathrm{P}\right]$-phosphohistone was prepared by the phosphorylation of bovine brain histone (MilliporeSigma, type-2AS) with cAMP-dependent protein kinase (PKA) in the presence of cAMP and [ $\left.{ }^{32} \mathrm{P}\right]$-ATP using established methods $(56,57)$. Phosphatase activity was measured by the quantitation of $\left[{ }^{32} \mathrm{P}\right]$-labeled orthophosphate liberated from the substrate using established protocols (57). 6,8-Difluoro-4-methylumbelliferyl phosphate-based (DiFMUP-based) inhibition assays were conducted as described $(56,57)$, in a 96-well format using DiFMUP (Invitrogen) $(100 \mu \mathrm{M}$ final assay concentration). $\mathrm{IC}_{50}$ values were calculated from a 10-point concentration/dose response curve by a 4-parameter logistic fit of the data, using 3-8 replicates per concentration.

Rib chondrocyte cultures. Rib cages were dissected from newborn mice ( $0-2$ days old) and trimmed to remove the skin, spinal cord, and soft tissue around the sternum and ribs. Nonchondrocyte tissue was digested away by incubating the rib cages in $2 \mathrm{mg} / \mathrm{mL}$ pronase (Roche, catalog 10165921001) in PBS for 1 hour in a shaking water bath at $37^{\circ} \mathrm{C}$ and was then incubated in $3 \mathrm{mg} / \mathrm{mL}$ collagenase $\mathrm{D}$ (Roche, catalog 11088866001) in medium for 1 hour. After washing, the rib cages were transferred to a dish with fresh collagenase $\mathrm{D}$ and incubated for 5-6 hours, with trituration at 2 hours, to release the chondrocytes. The isolated cells were passed through a $40 \mu \mathrm{m}$ nylon cell strainer (Corning, catalog 431750), resuspended in DMEM/F12 medium (Thermo Fisher Scientific, catalog 11320-033) with 10\% FBS (Thermo Fisher Scientific, catalog 10082-139), 100 units $/ \mathrm{mL}$ of penicillin, and $100 \mu \mathrm{g} / \mathrm{mL}$ of streptomycin. The cells were plated in $35 \mathrm{~mm}$ tissue culture dishes, at a density corresponding to 1 newborn mouse per dish, and cultured for 3 days, at which point the cells were approximately $75 \%-90 \%$ confluent. They were then washed with PBS and incubated in serum-free medium for 18 hours. The cells were then incubated in LB-100 $(10 \mu \mathrm{M})$, or control medium, followed by addition of FGF18 $(0.5 \mu \mathrm{g} / \mathrm{mL}+1 \mu \mathrm{g} / \mathrm{mL}$ heparin $)$ or control medium containing heparin only.

At end of the incubation period, dishes were washed in PBS, and cells were lysed in $250 \mu \mathrm{L}$ of $1 \%$ SDS containing $10 \mathrm{mM}$ sodium fluoride, $1 \mu \mathrm{M}$ microcystin-LR (Cayman Chemical, catalog 10007188), and protease inhibitor cocktail (Roche, catalog 04693159 001). Protein content was determined by a BCA assay (Pierce, catalog 23225). The protein yield per newborn mouse was approximately 200-300 $\mu$ g.

Phos-tag gel electrophoresis and Western blotting. Proteins were separated in a Phos-tag-containing gel, as previously described (58), except that chondrocyte lysates (30 $\mu \mathrm{g}$ protein) were used without immunoprecipitation. Phos-tag and protein size markers were obtained from Fujifilm Wako Pure Chemical (catalogs AAL-107 and 230-02461, respectively). For these studies, we used mice with HA-tagged NPR2 (43), and blots were probed with an antibody against the HA tag (Cell Signaling Technology, catalog 2367, 1:1000 dilution). The specificity of this antibody is validated in Supplemental Figure 3. Note that molecular weight markers are only approximate for Phos-tag gels.

Ex vivo culture of fetal femurs and skull base. Femurs from E16.5 embryos were cultured ex vivo, as described previously $(32,47)$. The left femur was cultured in the presence of LB-100 $(10 \mu \mathrm{M}), \mathrm{BMN}-111$ $(0.1 \mu \mathrm{M})$, or LB-100 $(10 \mu \mathrm{M})+\mathrm{BMN}-111(0.1 \mu \mathrm{M})$ and was compared with the vehicle-treated right femur. The bone's length was measured on day 0 (D0) and D6. Images were captured with an Olympus SZX12 stereo microscope and quantified using cellSens software (Olympus). The results were expressed as the increase in femur length or area (D6 - D0) in the presence or absence of LB-100, BMN-111, or LB-100 + BMN-111. Bone length and area were measured as shown in Supplemental Figure 5. To generate the graphs shown in Figure 3, the length or 2-dimensional area on D0 was subtracted from the length or area on D6 to calculate the amount of growth. These measurements of growth in drug-treated bones were divided by the mean values from corresponding measurements of control (vehicle-treated) bones; the graphs show the ratio of treated/control growth.

Embryonic skull base (E16.5) dissections were performed under an Olympus SZX12 stereo microscope and the skull bases (including the spheno-occipital and interoccipital synchondroses) were placed on top of $250 \mu \mathrm{L}$ of Matrigel (BD Biosciences) in 24-well plates and cultured for 6 days in DMEM with antibiotics and $0.2 \%$ BSA (MilliporeSigma) supplemented with vehicle or LB-100 $(10 \mu \mathrm{M})+$ BMN-111 $(0.1 \mu \mathrm{M})$. The distances between the BS, BO, and IO bones were measured on D0 and D6 using cellSens software (Olympus). Percentage increases in BS-BO and BO-IO were calculated for each sample by comparing D0 and D6. The mean of the left and right BO-IO measurements were used to calculate the BO-IO increase. Five embryos were used for each group.

Histology. After a 6-day culture period, fetal femur (E16.5) explants were fixed in 4\% paraformaldehyde, decalcified with EDTA (0.4M), and embedded in paraffin. Serial $5 \mu \mathrm{m}$ sections were stained with 
hematoxylin-eosin-safran (HES) reagent, using standard protocols. For immunohistochemical assessment, sections were labeled with the following antibodies and a Dako Envision Kit: anti-COLX (BIOCYC, catalog N.2031501005; 1:50 dilution), and anti-phosphorylated ERK1-2 (Thr180/Tyr182) (Cell Signaling Technology, catalog 4370; 1:100 dilution). Images were captured with an Olympus PD70-IX2-UCB microscope and quantified using cellSens software.

Mean areas of individual hypertrophic chondrocytes were measured from COLX-labeled sections, within a $166 \mu \mathrm{m}$ wide $\times 76 \mu \mathrm{m}$ high box positioned $50 \mu \mathrm{m}$ from mineralization front (Supplemental Figure 6). The measurements were made manually using Fiji software and the freehand selection tool. For analysis of the effect of the drug treatments on the area occupied by proliferative chondrocytes, these cells were identified by their round or columnar shape, as seen with HES staining, and by the absence of COLX labeling. We measured the total area occupied by chondrocytes within the whole growth plate and the area occupied by $\mathrm{COLX}{ }^{+}$chondrocytes. The area for proliferating chondrocytes was calculated by subtracting the $\mathrm{COLX}^{+}$area from the whole growth plate area.

Statistics. Data were analyzed using Prism 6 (GraphPad Software). To compare more than 2 groups, we used 1-way ANOVA followed by 2-tailed $t$ tests with the Holm-Sidak correction for multiple comparisons, or 2-way ANOVA followed by Sidak's multiple comparisons tests. Two groups were compared using either paired or unpaired 2-tailed $t$ tests, as indicated in the figure legends.

Study approval. All experiments were conducted as approved by the animal care committees of the University of Connecticut Health Center and the Imagine Institute, Université de Paris.

\section{Author contributions}

LCS, LAJ, and LLM designed the research and wrote the paper. NK performed the ex vivo bone growth experiments. MBD developed and analyzed the ex vivo skull base experiments. LCS and GV performed the cGMP imaging experiments. JRE, TFU, and TH performed the chondrocyte cell culture and Phos-tag analysis. LL and ED performed the immunolabeling quantitation. MRS and REH determined LB-100 selectivity for inhibition of PPP family phosphatases. LCS, NK, ED, MRS, MBD, and JRE prepared the figures.

\section{Acknowledgments}

This project received a state subsidy managed by the National Research Agency under the "Investments for the Future" program (ANR-10-IAHU-01). It was also supported by the NIH (R37HD014939 to LAJ and R01CA060750 to REH) and by the Fund for Science (to LCS and LAJ). We thank the Imagine Institute's imaging facility for their help with this work. We also thank Robert Feil for sharing the cGi500 mouse line; Deborah Kaback and Siu-Pok Yee for mouse colony management; Valentina Baena, Luisa Lestz, and Corie Owen for technical assistance; and Marja Hurley, Julian Lui, Florence Lorget, and Lincoln Potter for helpful discussions.

Address correspondence to: Laurinda A. Jaffe, Department of Cell Biology MC3636, University of Connecticut Health Center, 263 Farmington Ave., Farmington, Connecticut 06030, USA. Phone: 1.860.679.2661; Email: 1jaffe@uchc.edu. Or to: Laurence Legeai-Mallet, Université de Paris, Imagine Institute, Laboratory of Molecular and Physiopathological Bases of Osteochondrodysplasia, INSERM UMR 1163, F-75015, Paris, France. Phone: 33.42.75.43.02; Email: laurence.legeai-mallet@inserm.fr.

1. Rousseau F, et al. Mutations in the gene encoding fibroblast growth factor receptor-3 in achondroplasia. Nature. 1994;371(6494):252-254.

2. Shiang R, et al. Mutations in the transmembrane domain of FGFR3 cause the most common genetic form of dwarfism, achondroplasia. Cell. 1994;78(2):335-342.

3. Ornitz DM, Legeai-Mallet L. Achondroplasia: Development, pathogenesis, and therapy. Dev Dyn. 2017;246(4):291-309.

4. Colvin JS, et al. Skeletal overgrowth and deafness in mice lacking fibroblast growth factor receptor 3. Nat Genet. 1996;12(4):390-397.

5. Deng C, et al. Fibroblast growth factor receptor 3 is a negative regulator of bone growth. Cell. 1996;84(6):911-921.

6. Naski MC, et al. Repression of hedgehog signaling and BMP4 expression in growth plate cartilage by fibroblast growth factor receptor 3. Development. 1998;125(24):4977-4988.

7. Chen L, et al. Gly369Cys mutation in mouse FGFR3 causes achondroplasia by affecting both chondrogenesis and osteogenesis. J Clin Invest. 1999;104(11):1517-1525.

8. Li C, et al. A Lys644Glu substitution in fibroblast growth factor receptor 3 (FGFR3) causes dwarfism in mice by activation of STATs and ink4 cell cycle inhibitors. Hum Mol Genet. 1999;8(1):35-44.

9. Pannier S, et al. Delayed bone age due to a dual effect of FGFR3 mutation in Achondroplasia. Bone. 2010;47(5):905-915.

10. Lee YC, et al. Knock-in human FGFR3 achondroplasia mutation as a mouse model for human skeletal dysplasia. Sci Rep. 2017;7:43220.

11. Legeai-Mallet L, et al. Overexpression of FGFR3, Stat1, Stat5 and p21Cip1 correlates with phenotypic severity and defective 
chondrocyte differentiation in FGFR3-related chondrodysplasias. Bone. 2004;34(1):26-36.

12. Ornitz DM, Itoh N. The fibroblast growth factor signaling pathway. Wiley Interdiscip Rev Dev Biol. 2015;4(3):215-266.

13. Long F, Ornitz DM. Development of the endochondral skeleton. Cold Spring Harb Perspect Biol. 2013;5(1):a008334.

14. Maroteaux P, et al. Le nanisme acromésomélique. Presse Med. 1971;79(42):1839-1842.

15. Bartels CF, et al. Mutations in the trans-membrane natriuretic peptide receptor NPR-B impair skeletal growth and cause acromesomelic dysplasia, type Maroteaux. Am J Hum Genet. 2004;75(1):27-34.

16. Khan S, et al. Novel mutations in natriuretic peptide receptor-2 gene underlie acromesomelic dysplasia, type maroteaux. $B M C M e d$ Genet. 2012;13:44.

17. Miura K, et al. Overgrowth syndrome associated with a gain-of-function mutation of the natriuretic peptide receptor 2 (NPR2) gene. Am J Med Genet A. 2014;164A(1):156-163.

18. Bocciardi R, et al. Overexpression of the C-type natriuretic peptide (CNP) is associated with overgrowth and bone anomalies in an individual with balanced $t(2 ; 7)$ translocation. Hum Mutat. 2007;28(7):724-731.

19. Moncla A, et al. A cluster of translocation breakpoints in $2 \mathrm{q} 37$ is associated with overexpression of NPPC in patients with a similar overgrowth phenotype. Hum Mutat. 2007;28(12):1183-1188.

20. Chusho C, et al. Dwarfism and early death in mice lacking C-type natriuretic peptide. Proc Natl Acad Sci U S A. 2001;98(7):4016-4021.

21. Tamura N, et al. Critical roles of the guanylyl cyclase B receptor in endochondral ossification and development of female reproductive organs. Proc Natl Acad Sci US A. 2004;101(49):17300-17305.

22. Tsuji T, Kunieda T. A loss-of-function mutation in natriuretic peptide receptor 2 (Npr2) gene is responsible for disproportionate dwarfism in cn/cn mouse. J Biol Chem. 2005;280(14):14288-14292.

23. Geister KA, et al. A novel loss-of-function mutation in Npr2 clarifies primary role in female reproduction and reveals a potential therapy for acromesomelic dysplasia, Maroteaux type. Hum Mol Genet. 2013;22(2):345-357.

24. Nakao K, et al. The Local CNP/GC-B system in growth plate is responsible for physiological endochondral bone growth. Sci Rep. 2015;5:10554.

25. Miura, et al. An overgrowth disorder associated with excessive production of cGMP due to a gain-of-function mutation of the natriuretic peptide receptor 2 gene. PLoS One. 2012;7(8):e42180.

26. Robinson JW, et al. Dephosphorylation is the mechanism of fibroblast growth factor inhibition of guanylyl cyclase-B. Cell Signal. 2017;40:222-229.

27. Shuhaibar LC, et al. Dephosphorylation of the NPR2 guanylyl cyclase contributes to inhibition of bone growth by fibroblast growth factor. Elife. 2017;4;6:e31343.

28. Potter LR. Phosphorylation-dependent regulation of the guanylyl cyclase-linked natriuretic peptide receptor B: dephosphorylation is a mechanism of desensitization. Biochemistry. 1998;37(8):2422-2429.

29. Potter LR, Hunter T. Identification and characterization of the major phosphorylation sites of the B-type natriuretic peptide receptor. J Biol Chem. 1998;273(25):15533-15539.

30. Wagner BM, et al. Prevention of guanylyl cyclase-B dephosphorylation rescues achondroplastic dwarfism. JCI Insight. 2021;6(9):e 147832.

31. Yasoda A, et al. Overexpression of CNP in chondrocytes rescues achondroplasia through a MAPK-dependent pathway. Nat Med 2004;10(1):80-86.

32. Lorget F, et al. Evaluation of the therapeutic potential of a CNP analog in a Fgfr3 mouse model recapitulating achondroplasia. Am J Hum Genet. 2012;91(6):1108-1114.

33. Savarirayan R, et al. C-type natriuretic peptide analogue therapy in children with achondroplasia. N Engl J Med. 2019;381(1):25-35

34. Savarirayan R, et al. Once-daily, subcutaneous vosoritide therapy in children with achondroplasia: a randomised, double-blind, phase 3, placebo-controlled, multicentre trial. Lancet. 2020;39(10252):684-692.

35. D'Arcy BM, et al. The antitumor drug LB-100 is a catalytic inhibitor of protein phosphatase 2A (PPP2CA) and 5 (PPP5C) coordinating with the active-site catalytic metals in PPP5C. Mol Cancer Ther. 2019;18(3):556-566

36. Ho WS, et al. Pharmacologic inhibition of protein phosphatase-2A achieves durable immune-mediated antitumor activity when combined with PD-1 blockade. Nat Commun. 2018;9(1):2126.

37. Lai D, et al. PP2A inhibition sensitizes cancer stem cells to ABL tyrosine kinase inhibitors in BCR-AB ${ }^{\mathrm{L}}+$ human leukemia. Sci Transl Med. 2018;10(427):eaan8735.

38. Cui J, et al. Inhibition of PP2A with LB-100 enhances efficacy of CAR-T cell therapy against glioblastoma. Cancers (Basel). 2020;12(1):139.

39. Chung V, et al. Safety, tolerability, and preliminary activity of LB-100, an inhibitor of protein phosphatase $2 \mathrm{~A}$, in patients with relapsed solid tumors: an open-label, dose escalation, first-in-human, phase I trial. Clin Cancer Res. 2017;23(13):3277-3284.

40. Honkanen RE. Cantharidin, another natural toxin that inhibits the activity of serine/threonine protein phosphatases types 1 and 2A. FEBS Lett. 1993;330(3):283-286.

41. Swingle MR, Honkanen RE. Inhibitors of serine/threonine protein phosphatases: biochemical and structural studies provide insight for further development. Curr Med Chem. 2019;26(15):2634-2660.

42. Kinoshita E, et al. Separation and detection of large phosphoproteins using Phos-tag SDS-PAGE. Nat Protoc. 2009;4(10):1513-1521.

43. Baena V, et al. Cellular heterogeneity of the LH receptor and its significance for cyclic GMP signaling in mouse preovulatory follicles. Endocrinology. 2020;161(7):bqaa074.

44. Pannier S, et al. Activating Fgfr3 Y367C mutation causes hearing loss and inner ear defect in a mouse model of chondrodysplasia. Biochim Biophys Acta. 2009;1792(2):140-147.

45. Krejci $\mathrm{P}$, et al. Interaction of fibroblast growth factor and C-natriuretic peptide signaling in regulation of chondrocyte proliferation and extracellular matrix homeostasis. J Cell Sci. 2005;118(21):5089-5100.

46. Ozasa A, et al. Complementary antagonistic actions between C-type natriuretic peptide and the MAPK pathway through FGFR-3 in ATDC5 cells. Bone. 2005;36(6):1056-1064.

47. Jonquoy A, et al. A novel tyrosine kinase inhibitor restores chondrocyte differentiation and promotes bone growth in a gain-offunction Fgfr3 mouse model. Hum Mol Genet. 2012;21(4):841-851.

48. Murakami S, et al. Constitutive activation of MEK1 in chondrocytes causes Stat1-independent achondroplasia-like dwarfism 
and rescues the Fgfr3-deficient mouse phenotype. Genes Dev. 2004;18(3):290-305.

49. Loveday C, et al. Mutations in the PP2A regulatory subunit B family genes PPP2R5B, PPP2R5C and PPP2R5D cause human overgrowth. Human Molec Genetics. 2015;24(17):4775-4779.

50. Robinson JW, et al. Male mice with elevated C-type natriuretic peptide-dependent guanylyl cyclase-B activity have increased osteoblasts, bone mass and bone strength. Bone. 2020;135:115320.

51. Matsushita M, et al. Low bone mineral density in achondroplasia and hypochondroplasia. Pediatr Int. 2016;58(8):705-708

52. An J-S, et al. Inhibition of fibrotic changes in infrapatellar fat pad alleviates persistent pain and articular cartilage degeneration in monoiodoacetic acid-induced rat arthritis model. Osteoarthritis Cartilage. 2021;29(3):380-388.

53. Potter LR, Garbers DL. Dephosphorylation of the guanylyl cyclase-A receptor causes desensitization. J Biol Chem. 1992; 267(21):14531-14534

54. Thunemann M, et al. Transgenic mice for cGMP imaging. Circ Res. 2013;113(4):365-371.

55. Swingle MR, Honkanen RE. Development and validation of a robust and sensitive assay for the discovery of selective inhibitors for serine/threonine protein phosphatases PP1 $\alpha$ (PPP1C) and PP5 (PPP5C). Assay Drug Dev Technol. 2014;12(8):481-496.

56. Ni L, et al. High yield expression of serine/threonine protein phosphatase type 5 , and a fluorescent assay suitable for use in the detection of catalytic inhibitors. Assay Drug Dev Technol. 2007;5(5):645-653.

57. Swingle M, et al. Small-molecule inhibitors of ser/thr protein phosphatases: specificity, use and common forms of abuse. Methods Mol Biol. 2007;365:23-38.

58. Egbert JR, et al. Dephosphorylation and inactivation of NPR2 guanylyl cyclase in granulosa cells contributes to the LH-induced decrease in cGMP that causes resumption of meiosis in rat oocytes. Development. 2014;141(18):3594-3604. 